
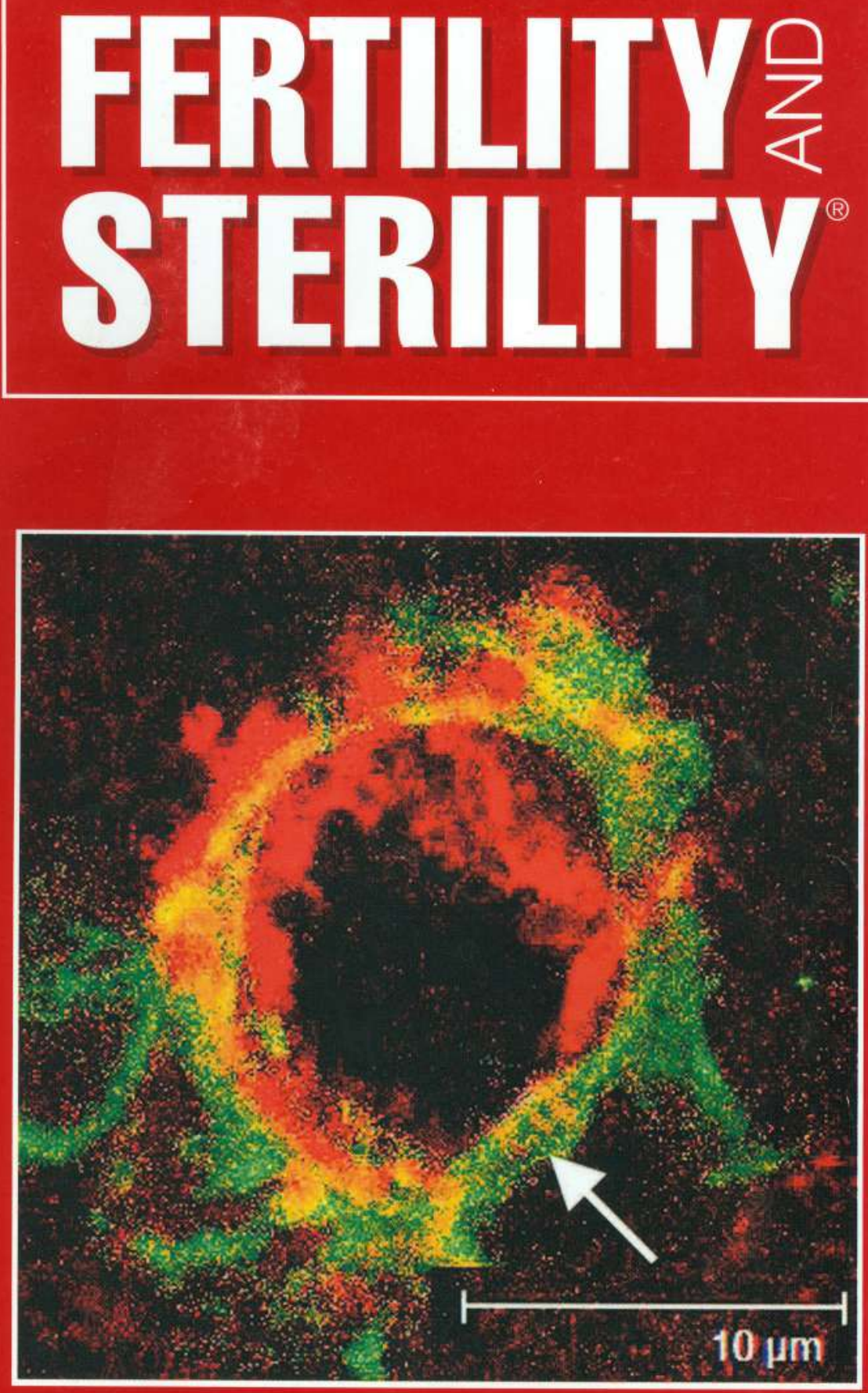

Submit Manuscripts Online at www.fertstert.org

The Official Journal of the American Society for

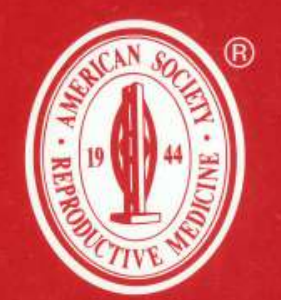




\title{
Growth differentiation factor 9 concentration in the follicular fluid of infertile women with endometriosis
}

\begin{abstract}
This study evaluated the level of growth differentiation factor 9 (GDF-9) in the follicular fluid of infertile women with endometriosis. The presence of GDF-9 in follicular fluid of preovulatory follicle was confirmed by Western blotting method in the band of $53 \mathrm{kDa}$, and GDF-9 concentration in women with severe endometriosis was lower than in those without. (Fertil Steril ${ }^{\circledR}$ 2010;94:758-60. (2010 by American Society for Reproductive Medicine.)
\end{abstract}

Endometriosis is a disorder characterized by the presence and growth of endometrial tissue in the ectopic site. Its prevalence among asymptomatic women ranges from $2 \%$ to $20 \%$, whereas in women with dysmenorrhoea the prevalence is $40 \%$ to $60 \%$ (1). It seems that the presence of endometriosis is associated with decreased oocyte quality. The reason is unclear (2). Several in vitro studies demonstrated that the growth and maturation of oocytes are dependent on nutrients from granulosa cells and growth factor from the oocyte itself (3). GDF-9 is a member of the transforming growth factor $\beta$ (TGF- $\beta$ ) superfamily. It is expressed at high levels in the mammalian oocyte beginning at the type 3 a primary follicle stage. In a mouse model, GDF-9 paracrine function is shown to be important for folliculogenesis. It induces granulosa cells to produce hyaluronan synthase 2 (HAS-2), which plays a role in hyaluran-dependent cumulus expansion (4). Other studies showed that GDF-9 activated androgen synthesis in theca cells and regulated meiotic competence (5).

We postulated that apoptosis of granulosa cells caused disturbance in oocyte growth and maturation and was associated with decreased GDF-9 production. The aim of the present study was to evaluate the level GDF-9 in the follicular fluid of infertile patients with endometriosis.

Hendy Hendarto, Ph.D. ${ }^{a}$

Prajitno Prabowo, M.D. ${ }^{\text {a }}$

Farid Anfasa Moeloek, Ph.D. ${ }^{c}$

Soetjipto Soetjipto, Ph.D. ${ }^{\text {b }}$

${ }^{a}$ Department of Obstetrics and Gynecology, Dr. Soetomo Hospital, Faculty of Medicine, University of Airlangga, Surabaya, Indonesia

${ }^{b}$ Lab of Biochemistry, Faculty of Medicine, University of Airlangga, Surabaya, Indonesia

${ }^{c}$ Department of Obstetrics and Gynecology, Dr. Cipto Mangunkusumo Hospital/Faculty of Medicine, University of Indonesia, Jakarta, Indonesia

Received May 8, 2009; revised October 2, 2009; accepted October 5, 2009; published online December 9, 2009.

H.H. has nothing to disclose. P.P. has nothing to disclose. F.A. has nothing to disclose. S.S. has nothing to disclose.

Supported by a Risbin Iptekdok grant from the Eijkman Institute of Molecular Biology, Jakarta, Indonesia.

Presented at the the World Congress on Endometriosis, Melbourne, Australia, March 11-14, 2008.

Reprint requests: Hendy Hendarto, Ph.D., Dept. of Obstetrics and Gynecology, Dr. Soetomo Hospital/School of Medicine, University of Airlangga, Surabaya, Indonesia, (FAX: 6231 5501704; E-mail: hndhendy@yahoo.com).
The study was conducted from November 2005 to March 2007 at Dr. Soetomo Hospital, Surabaya, Indonesia. The hospital's research and ethics board approved the study. Participants involved in the study were infertile women aged 20 to 35 years, with $(\mathrm{n}=31)$ and without endometriosis $(\mathrm{n}=19)$. Of those with endometriosis, 18 women had mild and 13 severe endometriosis. Staging of endometriosis was done using the revised classification of the American Society for Reproductive Medicine (6). Besides endometriosis, other infertility investigations were within normal limits. All patients had not taken any hormone medication at least 6 months before laparoscopy. Follicular fluid of preovulatory follicle was aspirated using a laparoscopic aspiration needle at the beginning of the laparoscopy.

Two research steps were conducted: step 1) characterization of GDF-9 protein by using Western blotting and deglycosylation methods; and step 2) detection of GDF-9 concentration by ELISA. Gel and nitrocellulose membrane were transfered. We used antibody anti-GDF-9 and antihuman IgG-alkaline phosphatase. Primary antibody incubation took place overnight at $4{ }^{\circ} \mathrm{C}$, and secondary incubation for 2 hours at room temperature. Detection of protein bands was performed by using chemiluminescence system. Ten millilters of phosphate buffer, $\mathrm{pH} 7.5$, and $2 \%$ sodium dodecyl sulfate (SDS) with mercaptoetanol were added to the protein sample which was then heated at $100^{\circ} \mathrm{C}$ for 5 minutes. To remove $\mathrm{N}$-linked oligosacharides, the follicular fluid was incubated at $37^{\circ} \mathrm{C}$ for 3 hours with Triton-X 100 and $15 \%$ peptide:N-glycosidase F (PNGase F; catalog no. G5166-50UN; Sigma Aldrich, Singapore) before Western blotting. For sham digestion, the follicular fluid was treated as for digestion but the enzyme was omitted. Protein was run by using SDS-polyacrylamide gel electrophoresis. One hundred microliters of phosphate-buffered saline-Tween20phenylmethylsulfonyl fluoride was added to the follicular fluid isolation sample. Primary antibody anti-GDF-9 (catalog no. AF739) and secondary antibody antirabbit IgG-alkaline phosphatase were used. GDF-9 concentration was measured with use of ELISA kit (R\&D Systems, Minneapolis, MN).

The results showed that the presence of GDF-9 in follicular fluid of preovulatory follicle was confirmed by Western blotting analysis in a band of $53 \mathrm{KDa}$. The PNGase F treatment of GDF-9-containing medium reduced the size of the $53 \mathrm{kDa}$ band to $48 \mathrm{kDa}$. Follicular fluid measurement using ELISA showed that the mean GDF-9 concentrations were $191.52 \pm 102.9 \mathrm{pg} / \mathrm{mL}$ in women with no endometriosis and $139.67 \pm 94.31 \mathrm{pg} / \mathrm{mL}$ in mild endometriosis, and $105.98 \pm 50.42 \mathrm{pg} / \mathrm{mL}$ in severe endometriosis. Analysis of variance was used, because the measurable variables were 
(A) Detection of protein with an approximate molecular weight of $53 \mathrm{kDa}$ (red arrow) in extracts from follicular fluid using anti-growth differentiation factor 9 (GDF-9) antibodies. The weakest color intensity is shown in lanes 2 and 3 , the strongest in lanes 6 and 7 . M = mild endometriosis; $\mathrm{N}=$ no endometriosis; $\mathrm{S}$ = severe endometriosis. (B) Deglycosylated (dG) GDF-9 using PNGase F. This treatment reduces the size of $53 \mathrm{kDa}$ band to $48 \mathrm{kDa}$. (C) Concentration $(\mathrm{pg} / \mathrm{mL})$ of GDF-9 in the follicular fluid by ELISA.
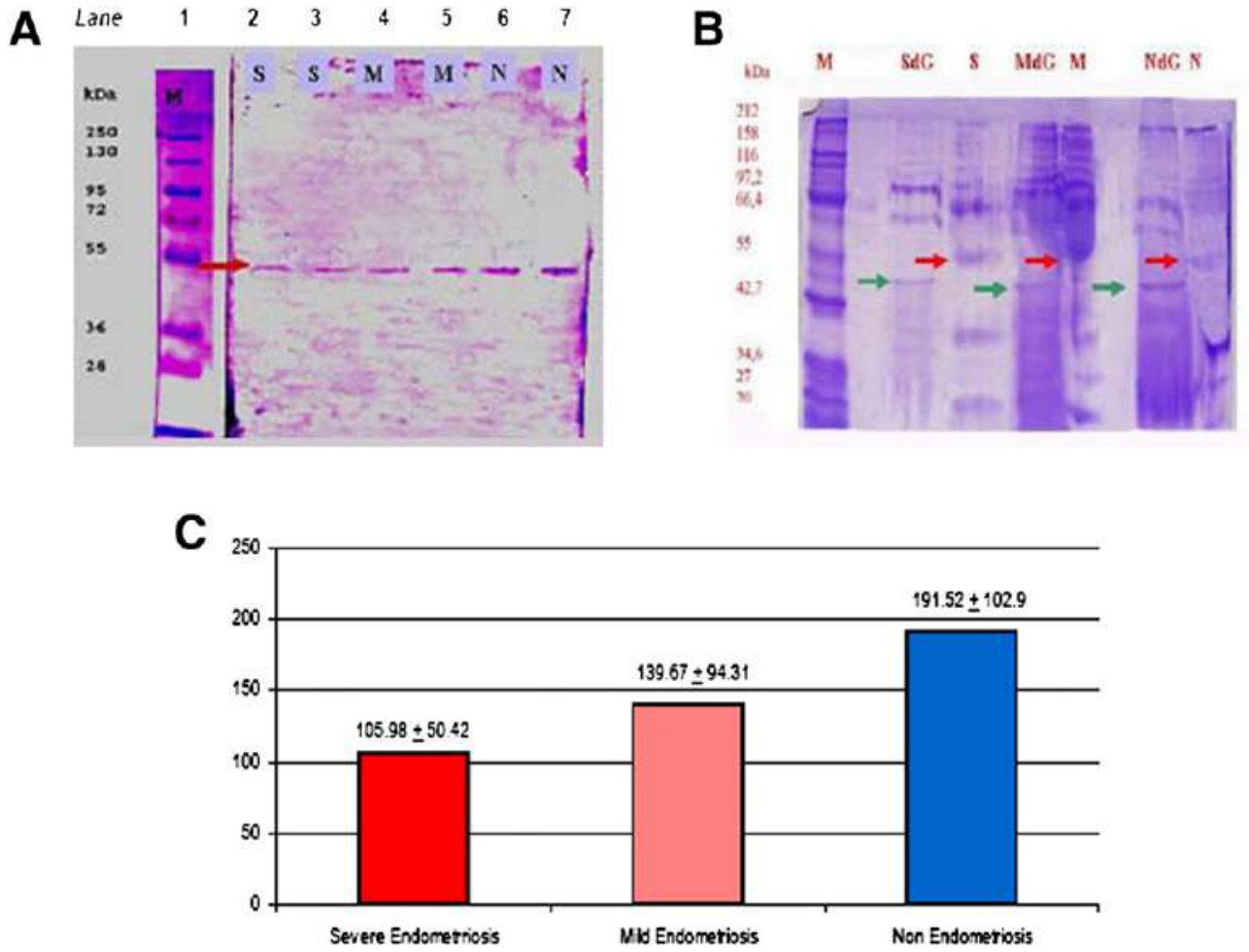

Hendarto. Correspondence. Fertil Steril 2010.

normally distributed. The results showed that there was a significant difference in concentration of GDF-9 among the no, mild, and severe endometriosis goups $(P=.03 ; 95 \%$ CI $21-150 \mathrm{pg} / \mathrm{mL})$. Additional exploration using post hoc least significant difference test showed that there was a significant difference in concentration of GDF-9 only between no and severe endometriosis $(P=.01)$. The same trend was seen by Western blotting method. The weakest color intensity was shown in lanes 2 and 3 (severe endometriosis). The strongest was in lanes 6 and 7 (no endometriosis). All subjects in each group actually had GDF-9 determinations. The range of GDF-9 concentrations were between 54.38 and 458.91 in no endometriosis, between 35.16 and 333.75 in mild endometriosis, and between 29,38 and 200.31 in severe endometriosis. All aspirated follicles were dominant follicles $>17 \mathrm{~mm}$ in diameter determined using transvaginal sonography before aspiration.

This study demonstrated the presence of GDF-9 in the follicular fluid of women with no endometriosis and with endometriosis. Compared with women with no endometriosis, the concentration was lower in those with severe endometriosis. Results are shown in Figure 1.

Growth differentiation factor 9 is a glycosylated protein. Carbohydrate in glycoprotein is important for intercellular communication. It is tightened to polypeptide chain via N-linked and O-linked oligosaccharide. To confirm N-linked glycosylation, we treated
GDF-9-containing medium with PNGase $F$ to remove the $\mathrm{N}$-linked oligosaccharides. This treatment reduced the size of the $53 \mathrm{kDa}$ band to $48 \mathrm{kDa}$. N-Glycosidase treatment of follicular fluid in rhesus monkey also yielded a shift in the apparent size of 54-MW band compared with undigested follicular fluid (7).

Previous studies have demonstrated expression of GDF-9 in normal human oocytes in primary follicles. The present study shows that these growth factors are also expressed in the follicular fluid of women with endometriosis.

In situ hybridiation study by Filho et al. (8) showed that the expression of GDF-9 was restricted to the oocyte in all ovaries examined. Interestingly, a decreased level of GDF-9 signal was observed in the developing follicles of women with polycystic ovary (PCO) syndrome and PCO oocytes compared with normal. Because oocyte-derived GDF-9 is crucial for normal folliculogenesis, we propose that dysregulation of oocyte GDF-9 expression may contribute to aberrant folliculogenesis in women with endometriosis. The present study is different from the PCO study by Filho et al. (8), where samples were taken from the follicular fluid by laparoscopy.

Growth differentiation factor 9 is synthesized in oocytes at all stages of the folliculogenesis process. Elvin et al. (4) reported that mouse GDF-9 can bind to receptors on granulosa cells, 
regulating the expression of several gene products. GDF-9 plays multifunctional roles in oocyte-granulosa cell communication and regulation of follicular differentiation and function (4). Culturing human ovarian biopsies with GDF-9 enhanced the progression of follicular maturation. Compared with the level in the follicular fluid of women with no endometriosis, we found that the GDF-9 level in those with severe endometriosis was lower. This low level may cause impairment of GDF-9 paracrine and autocrine function, impairing folliculogenesis and oocyte production.

Oocyte-secreted GDF-9 is currently the primary candidate molecule for the cumulus expansion-enabling factors. Dragovic et al. (9) demonstrated that GDF-9 can enable FSH-induced mouse cumulus expansion and have a paracrine action of oocyte on granu- losa or cumulus cells in vitro (9). The low level of GDF-9 in the follicular fluid of women with severe endometriosis might impair the cumulus expansion process and oocyte maturity. Indeed, in GDF-9-deficient mice, the absence of GDF-9 may cause abnormality of theca and granulosa cells that lead to infertility (10).

The present study demonstrated that compared the level in women with no endometriosis, GDF-9 level in the follicular fluid of women with severe endometriosis was lower. This might impair folliculogenesis, leading to decreased oocyte quality. Further study is needed.

Acknowledgments: The authors thank Prof. Togas Tulandi for his assistance in the preparation of the manuscript.

\section{REFERENCES}

1. Kyama CM, Debrock S, Mwenda JM D'Hooghe TM. Potential involvement of the immune system in the development of endometriosis: Review. Reprod Biol Endocrinol 2003;1:1-9.

2. Speroff L, Fritz MA. Endometriosis. In: Clinical gynecologic endocrinology and infertility. 7th ed. Philadephia: Lippincott Williams \& Wilkins, 2005:1103-33.

3. Briggs D, Miller D, Gosden R. Molecular biology of female gametogenesis. In: Fauser BCJM, Rutherford AJ, Strauss JF, Steirteghem AV, eds. Molecular biology in reproductive medicine. 1st ed. New York: Parthenon, 1999:251-70.
4. Elvin JA, Clark AT, Wang P, Wolfman NM, Matzuk M. Paracrine action of growth differentiation factor-9 in mammalian ovary. Mol Endocrinol 1999;13:1035-48.

5. Strauss JF, Lessey BA. The ovarian life cycle. In: Yen and Jaffe's reproductive endocrinology. 5th ed. Philadelphia: Elsevier Saunders, 2004:213-53.

6. American Society for Reproductive Medicine. Revised American Society for Reproductive Medicine classification of endometriosis. Fertil Steril 1997;67:817-21.

7. Duffy DM. Growth differentiation factor- 9 is expressed by the primate follicle throughout the periovulatin. Biol Reprod 2003;69:725-32.
8. Filho FLT, Baracat EC, Lee TH, Suh CS, Matsui M, Chang J, et al. Aberrant expression of growth differentiation factor-9 in oocytes of polycystic ovary syndrome. J Clin Endocrinol Metab 2001;87: 1337-44.

9. Dragovic RA, Ritter LJ, Schutz SJ, Amato F, Amstrong PT, Gilchrist RB. Role of oocyte-secreted GDF-9 in the regulation of cumulus expansion. Endrocinology 2005;146:2798-809.

10. Elvin JA, Yan C, Wang P, Nishimori K, Matzuk M. Molecular characterization of the follicle defects in the growth differentiation factor 9-deficient ovary. Mol Endocrinol 1999;13:1018-34. 